\title{
Translation and validation of communicative quality of life in the dysarthric speaker questionnaire in telugu
}

\author{
Philemon Benison Dasari', Himanshu Verma², G.V.M Hariprasad ${ }^{3}$ \\ 'Department of Speech Pathology \& Audiology, Ucchvas Rehabilitation Center, Telangana; ${ }^{2}$ Speech \& Hearing Rehabilitation Unit, Department of \\ Otolaryngology, Post Graduate Institute of Medical Education \& Research, Chandigarh; ${ }^{3}$ Department of Speech-Language Pathology, Ali Yavar Jung \\ National Institute of Speech \& Hearing Disabilities, Secunderabad, India
}

Purpose: Several tools are available to assess the quality of life for speech, language, voice, and swallowing disorders in Indian languages. However, limited tools are present to assess the quality of life of dysarthria. So, the present study aims to translate and validate the Quality of Life in the Dysarthric Speaker (QoL-DyS) questionnaire into the Telugu language.

Methods: A total of 30 different types of dysarthria and 30 age-matched controls participated in the present study. The QoL-DyS tool was trans-adapted in the Telugu language using a standardized procedure and administered to the participants. The internal consistency, test-retest reliability, construct validity, scale statistics, and item statistics were computed.

Results: The present study revealed that the developed tool has good (i.e., 0.81) overall internal consistency and excellent (i.e., 0.92) test-retest reliability. The significant difference (i.e., $p<0.05$ ) was found between the experimental and control group.

Conclusions: From the present study, we can conclude that the Telugu QOL-DyS is a reliable tool. It is applicable in clinical practice for the self-assessment of the quality of life in dysarthric speakers. Hence, the Telugu QOL-DyS is recommended in clinical practice, and there is a need to develop QoL-DyS in other Indian languages.

Keywords: Dysarthria, Quality of Life, Assessment tool

\section{INTRODUCTION}

Dysarthria is defined as a neurological motor speech disorder characterized by slow, weak, imprecise, and/or uncoordinated movements of the speech musculature [1]. It is often categorized in perceptually based types and/or according to a degree of dysarthria (mild, moderate, or severe) that reflect abnormalities in the strength, speed, range, steadiness, or accuracy of movements required for the breathing, phonation, resonation, articulation, or prosodic aspects of speech production. The responsible neurophysiologic disturbances of control or execution are due to one or more sensorimotor abnormalities, which often include weakness, spasticity, or excessive, reduced, or variable muscle tone. Duffy [2] advocated the use of dysarthria classification based on the site of lesion and characteristics. He classified the dysarthria as a flaccid, spastic, ataxic, hypokinetic, hyperkinetic, unilateral upper motor neuron, and mixed dysarthria based on the site of the lesion and characteristics.

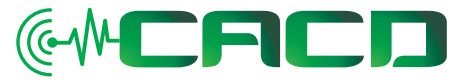

Received: October 20, 2021

Revision: December 23, 2021

Accepted: December 23, 2021

Correspondence:

Philemon Benison Dasari

Department of Speech-Language and Swallowing Pathology, Ucchvas Rehabilitation Center, 8-2-293/82/L, 350-A, Rd Number 12, Banjara Hills, Hyderabad, Telangana 500034, India Tel: +91-8186806040 E-mail: Benisonoz.csi@gmail.com

(C) 2021 The Korean Association of SpeechLanguage Pathologists

This is an Open Access article distributed under the terms of the Creative Commons Attribution NonCommercial License (https://creativecommons.org/ licenses/by-nc/4.0/) which permits unrestricted noncommercial use, distribution, and reproduction in any medium, provided the original work is properly cited. 
Sarno \& Levita [3] investigated the speech and language characteristics of patients with closed head injury with a history of coma. They reported that the majority of subjects manifest motor speech impairment along with linguistic processing deficits. Kent et al. [4] studied speech intelligibility and its phonetic \& acoustic correlates in a group of 10 women with amyotrophic lateral sclerosis (ALS). They compared the data for women with ALS, men with ALS with the data of the healthy geriatric control group. Results revealed a similar overall ranking of errors for males and females with ALS. However, males were more likely to have impairments of voicing in syllable-initial position. Tuner \& Weimar [5] studied the ability to alter speaking rate in a group of 9 subjects with ALS and nine age- and gender-matched, neurologically intact controls. Results suggested that the function relating physical to perceived speaking rate grew more rapidly for dysarthric than normal speakers. The above-stated speech issues in the dysarthria lead to poor speech intelligibility, and that person will not communicate adequately. Inadequate communication leads to many psychological issues which impaired the quality of life. The World Health Organization (WHO) defines the quality of life as individuals' "perception of their position in life in the context of the culture and value systems in which they live and in relation to their goals, expectations, standards, and concerns" (WHOQOL Group, 1995). ASHA defines the quality of communication life as "...the extent to which a person's communication acts, as constrained within the boundaries drawn by personal and environmental factors, and as filtered through this person's perspective, allow meaningful participation in life situations" [6]. The quality of life assessment plays a significant role in providing the patients' perspective about their health status.

In healthcare measurements, investigation of patent's health-related QOL is more recently used to make the clinical decision-making [7]. In the field of language, voice, and swallowing disorders, several QOL assessment tools were developed, e.g., the voice handicapped Index [8], voice-related quality of life [9], Aachen life quality inventory [10] to assess the quality of life of stroke patients with aphasia, communication effectiveness survey for dysarthria and idiopathic Parkinson disease [11]. In 2011, Piacentini et al. [12] developed the 40 item questionnaire to measure the quality of life in the dysarthric speakers (QoL-DyS). This questionnaire is easy to use and will provide better insight into the problems faced by dysarthric speakers due to their speech impairment. This questionnaire was originally developed in Italian and translated into different European languages (e.g., Nogueira et al. [13]) due to its clinical implications. To our best knowledge, it is not adapted in any Indian languages so, the present study aims to translate and validate the Qol-DyS questionnaire into the Telugu language.

\section{METHODS}

\section{Participants}

A total of 60 subjects participated in the present study. The subjects were divided into two groups, i.e., experimental \& control groups. The experimental group consists of 30 dysarthric subjects with a mean age of 45.65 years, as shown in Table 1 . The control group includes 30 age-matched healthy individuals with a mean age of 41.23 years.

\section{Inclusion \& Exclusion Criteria}

We included the native speakers of the Telugu language above 15 years of age with intact reading abilities. The participants had to undergo the screen of cognitive functions to rule out cognitive decline using the Montreal Cognitive Assessment (Naserddine, 1995). The present study included subjects from any origin and severity of dysarthria.

\section{Translation and Validation of the QoL-DyS Instrument}

Translation and validation were executed into two phases. In the first phase, translation and linguistic adaptation were performed, and during the second phase, the translated final questionnaire was administered to the subjects to validate the Telugu version of QoL-DyS.

\section{Phase I}

The translation of the QoL-DyS questionnaire was done using a standardized procedure, i.e., forward-backward translation method. The English version of the questionnaire was given

Table 1. Types of dysarthria included in the present study

\begin{tabular}{ll}
\hline Number of Subjects & Type of Dysarthria \\
\hline 3 & Flaccid \\
6 & Spastic \\
6 & Hyperkinetic \\
3 & Hypokinetic \\
2 & Ataxic \\
4 & Mixed dysarthria \\
6 & Unilateral upper motor neuron \\
\hline
\end{tabular}


to the three Telugu professors with equally proficient in English. All three Telugu translated versions were combined into a single questionnaire by the authors. The translated questionnaire was given for backward translation to the two individuals with equal proficiency in Telugu and English. The final Telugu version of the QoL-DyS questionnaire was given to the two experienced speech-language pathologists and the one linguist. The feedback and suggestions given were taken into consideration, and the final Telugu version of QoL-DyS was developed.

Prior to collecting the data, a pilot study was conducted on five healthy individuals and five people with dysarthria. A pilot study was conducted to assess the comprehensiveness, length, adequacy, redundancy, and text clarity. Based on the pilot study, nine more questions were added in the developed Telugu QoL-DyS tool from the 100 questions developed by Yorkston et al. 1994. So final tool consisted of two parts:

Part 1: Consist of all the first six questions in all the four subcategories

Part 2: Consist of all the remaining questions in all the four subcategories

\section{Phase II}

Before collecting the data, the aim and objectives of the study were explained to the participants, and informed consent was taken. The final developed Telugu version of the QoL-Dys questionnaire was given to the subjects and asked to fill the questionnaire.

\section{Data Analysis}

The data analysis was done using the SPSS 20.00 version software. The descriptive analysis was performed to compute the mean and standard deviation. The internal consistency of the tool was obtained using the Spearman-Brown Coefficient. To assess the difference between the responses of the experimental and control groups z-test was used. The test-retest re- liability was assessed using the Intra-class correlation coefficient (ICC).

\section{RESULTS}

\section{Internal Consistency of the Telugu QoL-DyS questionnaire}

The overall internal consistency of the tool was assessed using the Spearman-Brown Coefficient, and a test revealed good (i.e., 0.81 ) overall internal consistency. The analysis revealed 0.92 (excellent) \& 0.88 (good) internal consistency for the part I \& II respectively as shown in Table 2.

\section{Test-Retest Reliability}

The 15 subjects were retested after one week of the first assessment. The ICC was computed to assess the test-retest reliability and revealed excellent (i.e., 0.92) test-retest reliability.

\section{Construct Validity}

The z-test was used to assess the construct validity of the Telugu QoL-DyS tool and revealed a significant difference (i.e., $p<0.05$ ) between the experimental and control group.

\section{Item statistics}

As shown in Table 3, values of response elicited for the questions on a 5 point Likert rating scale of the questionnaire are:

Table 2. Represents the internal consistency of the Telugu QoL-DyS tool

\begin{tabular}{llll}
\hline Reliability Statistics & & & \\
\hline Cronbach's Alpha & Part 1 & Value & .921 \\
& & N of Items & 25 \\
& Part 2 & Value & .875 \\
& & N of Items & 24 \\
& Total N of Items & 49 \\
Correlation Between Forms & & .680 \\
Spearman-Brown Coefficient & Equal Length & .810 \\
& Unequal Length & .810 \\
Guttman Split-Half Coefficient & & .805 \\
\hline
\end{tabular}

Table 3. Represents the summary of item statistics

\begin{tabular}{|c|c|c|c|c|c|c|c|c|}
\hline & & \multicolumn{7}{|c|}{ Summary Item Statistics } \\
\hline & & Mean & Min & Max & Range & Max/Min & Variance & $\mathrm{N}$ of Items \\
\hline \multirow[t]{3}{*}{ Item Means } & Part 1 & 2.552 & 1.567 & 3.333 & 1.767 & 2.128 & .300 & 25 \\
\hline & Part 2 & 2.738 & 1.567 & 3.400 & 1.833 & 2.170 & .293 & 24 \\
\hline & Both Parts & 2.643 & 1.567 & 3.400 & 1.833 & 2.170 & .299 & 49 \\
\hline
\end{tabular}


Table 4. Demonstrates the results of the scale statistics

\begin{tabular}{lcccc}
\hline & \multicolumn{4}{c}{ Scale Statistics } \\
\cline { 2 - 5 } & Mean & Variance & $\begin{array}{c}\text { Std. } \\
\text { Deviation }\end{array}$ & N of Items \\
\hline Part 1 & 63.80 & 172.097 & 13.119 & 25 \\
Part 2 & 65.70 & 129.666 & 11.387 & 24 \\
Both Parts & 129.50 & 504.948 & 22.471 & 49 \\
\hline
\end{tabular}

Part 1: consists of 25 items; the overall mean value is 2.552 , the minimum value is 1.567 , the maximum value is 3.333 , and the range found to be 1.767 .

Part 2: consists of 24 items; the overall mean value is 2.738, the minimum value is 1.567 , the maximum value is 3.400 , and the range found to be 1.767 .

\section{Scale Statistics}

As shown in Table 4, scale statistical analysis revealed the rating of all the questions on a five-point Likert scale is as follows:

Part 1: consists of 25 items; the overall mean value is 63.80 , and the standard deviation is $\mathbf{1 3 . 1 1 9}$

Part 2: consists of 24 items; the overall mean value is 65.70, and the standard deviation is 11.387 .

Overall, part 1 and part 2 together consist of 49 items, and their mean value is 129.50 , and the standard deviation is 22.471 .

\section{DISCUSSION}

From past literature, we can conclude that many assessment tools to assess the quality of life of different communication disorders were developed. However, there was a lack of quality of life assessment tools for the dysarthric population. However, many assessment procedures have been developed to identify dysarthria. So, the present study aimed to develop a quality of life assessment tool for the Telugu dysarthric population.

The results of the present study revealed good internal consistency and test-retest reliability. The same results were also reported by Nogueira et al. (2019). They translated the QoLDyS tool into the European Portuguese language. The patients efficiently completed the QOL-DyS questionnaire and showed a strong internal consistency and reliability; furthermore, it presented scores significantly higher in dysarthric patients than in a control group. The study shows that the TQOLDyS is a valuable tool to assess perceived quality of life in adult patients with dysarthria. Questionnaire development was based on previous work by Yorkston et al. 2004; even if the original questionnaire has never been validated, it is considered a comprehensive tool and has been used to validate other instruments. From the original developed Trans adapted questionnaire, some of the questions were not easily understood by the patients: e.g.: questions belonging to situational difficulty: తందరగావేలేసి హారో మీరు మాట్లో డనప్ము د.....('I have difficulty speaking when I am in a hurry') and some other questions related to the same category. However, later questions were easily understood by filling all those situational difficulty category questions. This confusion made the patients more focused on what kind of questions they were filling instead of simply marking the most severe number. The original questionnaire of 40 items lacked some Telugu-speaking community-based questions to address and find the exact trouble in all the four subcategories. During the Pilot study, experts suggested nine more questions to fulfill the missing questions that address the problems of Telugu speaking community from the 100 questions developed by Yorkston et al. 1994 from the questionnaire entitled 'Dysarthria from the Point of View of the Dysarthric Patients.' Most of the patients included in the item study did not complain of any fatigue or discouragement while filling the questionnaire. In fact, Telugu QOL-DyS was easily completed and "very comprehensive according to their feedback when asked are these questions difficult to understand and rate". Application of the Telugu QOLDyS in different populations of patients with dysarthria showed different scores in the different populations.

Interestingly, when the questionnaire is segregated into four subcategories the severity scores in the four subcategories were not equally distributed in the different types of dysarthria. For example, patients with a unilateral upper motor neuron (UUMN) dysarthria scored higher in four subcategories. Whereas ataxic dysarthrics scored significantly lower, this shows how the quality of life with UUMN was more affected than ataxic dysarthrics. Those with flaccid dysarthria scored lower, similar to ataxic dysarthrics in perceived reactions of others and compensatory strategies subcategories. The scores were higher in situational difficulty and speech characteristics of word, which are similar to a hyperkinetic, hypokinetic, 
spastic, and mixed dysarthrics. The differences suggest the possibility that varied Telugu QOL-DyS profiles exist in different types of dysarthric speakers. The overall internal consistency of the TQOL-DyS was good. In two parts, the internal consistency ranged from good to excellent values considered acceptable for group comparison. Similar findings were reported in the validation of other QOL instruments [9, 14-16].

\section{CONCLUSIONS}

From the present study results, we can conclude that the reliability of the developed tool was good based on the spearman brown coefficient for the Telugu QOL-DyS questionnaire. It supports the application of the Telugu QOL-DyS. The scores obtained in dysarthric patients were significantly higher than those found in the control group when analyzed with the " $\mathrm{Z}$ " test. However, in the control group, compensatory strategies are present. There is a drastic difference between overall Telugu QOL-DyS scores and the results of an experimental group when compared with a control group. In conclusion, the Telugu QOL-DyS is reliable and appears easily applicable in daily practice for the self-assessment of the quality of life in dysarthric speakers. Hence, the Telugu QOL-DyS is recommended in clinical practice, and there is a need to develop QoL-DyS in other Indian languages.

\section{REFERENCES}

1. Darley FL, Aronson AE, Brown JR. Clusters of deviant speech dimensions in the dysarthrias. Journal of Speech, Language, and Hearing Research. 1969;12:462-496

2. Duffy JR. Motor Speech Disorders: Clues to Neurologic Diagnosis. In: Adler C.H., Ahlskog J.E. (eds) Parkinson's Disease and Movement Disorders. Current Clinical Practice. Humana Press, Totowa, NJ. 2000.

3. Sarno MT, Buonaguro A, Levita E. Characteristics of verbal impairment in closed head injured patients. Archives of Physical Medicine and Rehabilitation. 1986;67:400-405.

4. Kent JF, Kent RD, Rosenbek JC, Weismer G, Martin R, Sufit R, Brooks BR. Quantitative description of the dysarthria in women with amyotrophic lateral sclerosis. Journal of Speech, Language, and Hearing Research. 1992;35:723-733.
5. Turner GS, Weismer G. Characteristics of speaking rate in the dysarthria associated with amyotrophic lateral sclerosis. Journal of Speech, Language, and Hearing Research. 1993;36:1134-1144.

6. Paul DR, Frattali CM, Holland AL, Thompson CK, Caperton CJ, Slater SC. Quality of Communication Life Scale, Rockville, MD: The American Speech-Language-Hearing Association. 2004.

7. Testa MA, Drescher S: Assessment of qualityof-life outcomes. N Engl J Med. 1996;343:835- 840

8. Jacobson BH, Johnson A, Grywalski C, Silbergleit A, Jacobson G, Benninger MS, Newman CW. The Voice Handicap Index (VHI): development and validation. Am J Speech Lang Pathol. 1997;6: 66-70.

9. Hogikyan ND, Sethuraman G. Validation of an instrument to measure voice- related quality of life (V-RQOL). Journal of voice. 1999;13:557-569.

10. Engell B, Hütter B, Willmes K, Huber W. Quality of life in aphasia: Validation of a pictorial self-rating procedure, Aphasiology. 2003;17:383-396.

11. Donovan NJ, Kendall DL, Young ME, Rosenbek JC. The communicative effectiveness survey: Preliminary evidence of construct validity. American Journal of Speech-Language Pathology. 2008;17:335-347.

12. Piacentini V, Zuin A, Cattaneo D, Schindler A. Reliability and validity of an instrument to measure quality of life in the dysarthric speaker. Folia phoniatrica et logopaedica : official organ of the International Association of Logopedics and Phoniatrics (IALP). 2011;63:289-295.

13. Nogueira D, Reis E, Ferreira P, Schindler A. Measuring Quality of Life in the Speaker with Dysarthria: Reliability and Validity of the European Portuguese Version of the QoL-DyS. Folia phoniatrica et logopaedica : official organ of the International Association of Logopedics and Phoniatrics (IALP). 2019;71:176-190.

14. McHorney CA, Robbins J, Lomax K, Rosenbek JC, Chignell K, Kramer AE, Bricker DE. The SWAL-QOL and SWAL-CARE outcomes tool for oropharyngeal dysphagia in adults: III. Documentation of reliability and validity. Dysphagia. 2002;17:97-114.

15. Chen AY, Frankowski R, Bishop-Leone J, Hebert T, Leyk S, Lewin J, et al. The development and validation of a dysphagia-specific quality-of-life questionnaire for patients with head and neck cancer: the MD Anderson dysphagia inventory. Archives of otolaryngology-head \& neck surgery. 2001;127:870-876.

16. Woisard V, Andrieux MP, Puech M. Validation of a self-assessment questionnaire for swallowing disorders (Deglutition Handicap Index). Revue de laryngologie-otologie-rhinologie. 2006;127:315325. 